\title{
Déficit en antigènes d'histocompatibilité de classe I et transporteur de peptides
}

Les molécules d'histocompatibilité (HLA) de classe I sont des molécules exprimées à la surface des cellules et formées de l'association d'une chaîne lourde polymorphe, de la $\beta 2$-microglobuline $(\beta 2 \mathrm{~m})$ et d'un peptide provenant de la dégradation de protéines cytosoliques. Lorsque ce peptide dérive d'antigènes tumoraux ou d'agents infectieux intracellulaires, les molécules d'histocompatibilité interagissent avec les récepteurs de lymphocytes $\mathrm{T}$ spécifiques $\alpha \beta$ cytotoxiques CD8+. Par cette interaction, les cellules sont reconnues comme exprimant des protéines étrangères et sont lysées. Les molécules de classe I jouent donc a priori un rôle primordial dans la défense immunitaire et on pourrait s'attendre à ce qu'un déficit en molécules de classe I soit létal. Ce n'est pourtant pas le cas : quelques rares cas de déficit humains d'expression en antigènes de classe I ont été observés (syndrome du lymphocyte nu de type I). Sur le plan clinique, trois types de patients ont été décrits. Certains présentent un tableau d'insuffisance respiratoire résultant, semble-t-il, d'une colonisation bactérienne. D'autres plus sévèrement atteints souffrent de diarrhées fréquentes et d'infections bactériennes pulmonaires et meurent au cours de la jeune enfance. Enfin, il a été rapporté le cas de deux frères, âgés de 6 et 9 ans, qui ne présentaient pas de signes cliniques d'immunodéficience. Paradoxalement, les déficits ne se traduisent donc pas par une susceptibilité accrue aux infections virales. Cette diversité clinique est probablement corrélée à une hétérogénéité biologique. Une analyse plus détaillée montre, en effet, que certains de ces cas ne présentent
I, alors que d'autres, les plus sévères, pourraient présenter un déficit combiné en molécules de classe I et de classe II.

Nous venons de décrire une famille dont deux enfants présentent un déficit d'expression en molécules de classe I [1]. L'un d'eux, une jeune fille âgée de 16 ans, est suivie médicalement en raison d'une obstruction pulmonaire persistante ne répondant pas aux bronchodilatateurs et d'une colonisation chronique par Hemophilus influenza et par du pneumocoque. Son frère, âgé de 6 ans, en bonne santé, s'est révélé ne pas exprimer, lui non plus, les molécules de classe I lors du typage HLA de la famille.

Pour comprendre la nature du déficit, il peut être utile de rappeler par quels mécanismes les molécules de classe I présentent des peptides à la surface cellulaire. Dans une cellule normale, les chaînes lourdes des molécules de classe I s'associent à la $\beta 2 \mathrm{~m}$ dans la lumière du réticulum endoplasmique (RE). Ce complexe binaire y reste localisé, maintenu par des molécules chaperons, jusqu'à ce qu'un peptide approprié se fixe dans une niche prévue à cet effet et localisée sur la chaîne lourde. La fixation du peptide stabilise les molécules de classe I dans une conformation qui leur permet de quitter le RE. Le complexe ternaire transite alors par l'appareil de Golgi, où la chaîne lourde acquiert une glycosylation complexe, puis migre vers la surface cellulaire.

Les peptides que fixent les molécules de classe I sont produits dans le cytosol et résultent de la dégradation des protéines intracellulaires par des complexes multiprotéiques, les protéasomes. Il a été démontré récem- ment qu'un constituant de ce complexe, la sous-unité LMP7, est directement impliqué dans la genèse de certains peptides présentés par les molécules de classe I $\left(\mathrm{m} / \mathrm{s} n^{\circ} 10\right.$, vol. 10, p. 1059 [2]). Ces peptides sont ensuite transférés dans la lumière du RE par un transporteur de peptides, formé de deux sous-unités, dénommées TAPl et TAP2 (TAP pour transporter associated to antigen processing, pour revue voir [3]). Des lignées cellulaires déficitaires en transporteur de peptides ont été obtenues. Dans ces cellules, les chaînes lourdes des molécules de classe I s'associent à la $\beta 2 \mathrm{~m}$, mais, en l'absence de peptide, restent pour la plupart bloquées dans le RE. Quelques molécules s'en échappent cependant et réussissent à gagner la surface cellulaire où elles sont instables car exemptes de peptide. Ces cellules expriment peu de molécules de classe I et, en général, ne sont pas la cible de lymphocytes cytotoxiques CD8+.

Le déficit d'expression en antigènes de classe I peut donc s'expliquer par un défaut de transcription de l'ensemble des gènes de classe I, par une absence de $\beta 2 \mathrm{~m}$, ou encore par l'absence de peptide consécutive à une mutation dans une sous-unité du transporteur de peptides. Dans les deux cas rapportés, les deux premières hypothèses ont pu être rejetées : les ARNm codant pour les chaînes lourdes des molécules d'histocompatibilité sont normalement présents dans les lymphocytes et la $\beta 2 \mathrm{~m}$ est détectée dans les urines. Un déficit en transporteur de peptides a donc été envisagé.

Les gènes des transporteurs de peptides étant localisés à proximité des gènes d'histocompatibilité de classe II, nous avons d'abord recher- 
ché si le défaut ségrégeait avec les haplotypes HLA. De fait, le typage HIA de la famille a montré que les parents, cousins au premier degré, ont en commun un haplotype HLA et que les deux membres de la famille homozygotes pour l'haplotype HIA commun, sont justement les deux enfants qui n'expriment pas les molécules de classe I. Une étude biochimique et génétique plus complète a ultérieurement mis en évidence que les deux sujets sont homozygotes pour une mutation stop dans le gène de la sous-unité TAP2, mutation qui entraîne l'inactivation du transporteur de peptides.

Les lignées cellulaires établies à partir des lymphocytes B des patients ont les mêmes propriétés que les lignées déficitaires en transporteur de peptides obtenues par mutagenèse. Ainsi, dans ces cellules, les molécules de classe I HLA-A, -B ou -C ne sont pas chargées en peptides. Elles demeurent de ce fait instables, transitent avec inefficacité vers la surface cellulaire et peu de molécules sont exprimées en surface (moins de $3 \%$ par rapport à des cellules normales). En revanche, les molécules $\mathrm{CD} 1$, molécules non polymorphes constituées comme les molécules HI A-A, -B ou $-C$ d'une chaîne lourde $\alpha$ et de la $\beta 2 \mathrm{~m}$, sont exprimées sur les cellules cutanées de Langerhans et les cellules dendritiques différenciées à partir des monocytes des deux sujets [ 1 , 4]. Ces molécules, proches des molécules de classe I par leur structure, peuvent donc être présentes en surface indépendamment du transporteur de peptides.

La maturation des lymphocytes $T \alpha \beta$ cytotoxiques $\mathrm{CD} 8+$ requiert l'interaction de leurs récepteurs $\mathrm{T}$ avec les molécules de classe I présentes à la surface des cellules thymiques. Ainsi, les souris transgéniques qui n'expriment pas la $\beta 2 \mathrm{~m}$ ou le transporteur de peptides, et qui sont de ce fait déficitaires en molécules de classe I, n'ont qu'un nombre très faible de tels lymphocytes en périphérie. A l'inverse, les deux patients que nous avons décrits ont développé un nombre faible mais substantiel de telles cellules (5 à $10 \%$ des lymphocytes $\mathrm{T}$, comparativement à $>30 \%$ chez un individu normal). Ces lym- phocytes, capables d'exercer une activité cytotoxique allogénique chez l'un des enfants, peuvent être considérés comme mûrs.

Par ailleurs, la jeune fille qui présente une symptomatologie pulmonaire marquée a développé un nombre très important de lymphocytes $\mathrm{T} \gamma \delta$ (30\% des lymphocytes T, contre 5 à $10 \%$ pour un sujet sain). Ce n'était pas le cas de son jeune frère, bien portant quand l'étude a commencé, et qui avait un nombre normal de tels lymphocytes lors des premières investigations. Depuis, cet enfant à développé une maladie pulmonaire semblable à celle de sa sour et nous avons pu observer une expansion importante de cellules $\mathrm{T} \gamma \delta$.

La pathologie liée au déficit en molécules de classe I décrit ici est difficile à comprendre. On s'attendrait à ce que les patients présentent des infections virales sévères, et non des infections bactériennes pulmonaires. L'absence de transporteur de peptides entraîne, en effet, une non-présentation des antigènes intracellulaires. Ce défaut de présentation des antigènes viraux a été vérifié in vitro au moyen de lymphocytes $T$ cytotoxiques HLA-compatibles avec les cellules des patients. Or les patients ont bien été en contact avec des agents viraux ; leurs sérums contiennent des anticorps contre les virus d'Epstein-Barr, de l'herpès, du cytomégalovirus, de la rougeole, des oreillons ou de la grippe, sans qu'il ait pour autant été noté de maladie virale particulièrement sévère. Il faut donc admettre l'importance d'autres voies de défense qui ne seraient pas fondées sur la présentation antigénique relayée par le transporteur de peptides. On peut envisager, en premier lieu, une présentation de peptides par les molécules de classe I, indépendante du transporteur de peptides. Une telle présentation antigénique a déjà été observée in vitro avec les lignées déficitaires en transporteur. Il a été montré récemment que les protéines dites de "choc thermique" peuvent intervenir dans cette voie qui semble impliquer un transport des antigènes présentés vers des compartiments de dégradation endosomique $\left(m / s \quad n^{\circ} 6-7, \mathrm{vol}\right.$. 10, p. 719) [5]. Cependant, bien qu'il existe de plus en plus d'exemples de présentations indépendantes des transporteurs de peptides, ces exemples restent marginaux [6]. En second lieu, les lymphocytes CD4+ cytotoxiques pourraient jouer un rôle important, comme cela a été établi lors d'infections virales chez des souris déficitaires en $\beta 2 \mathrm{~m}$. L'interféron $\gamma$, connu pour induire l'expression des molécules de classe II sur la plupart des types cellulaires, et les protéines de choc thermique, dont on vient de montrer qu'elle peuvent permettre le transport de protéines intracellulaires vers les endosomes [5], pourraient relayer ce processus. Enfin les cellules NK pourraient être impliquées dans la réponse antivirale bien que les lymphocytes des deux sujets n'exercent aucune activité cytotoxique contre les cellules K562 qui, dépourvues d'antigènes de classe I, sont classiquement employées pour tester l'activité des cellules NK. Ces cellules pourraient, en effet, être activées localement par la libération de cytokines comme l'IL-2 ou l'interféron $\alpha$, puis exercer une activité cytotoxique antivirale. Cette hypothèse doit être retenue, car un déficit complet en cellules NK aboutit à des maladies inf ectieuses virales sévères, maladies non observées chez les deux enfants. Enfin, les réponses relayées par les anticorps pourraient avoir un rôle clé, soit en déclenchant une cytotoxicité relayée par le complément, soit en permettant des réponses relayées par les cellules effectrices comme les monocytes/macrophages, ou les cellules NK. Ces réponses induiraient la production locale de cytokines nécessaires à l'activation des réponses précédemment suggérées.

Les mécanismes immunitaires envisagés précédemment, ne sont probablement pas aussi rapides que le déclenchement des réponses relayées par les cellules $\mathrm{T}$ CD8t. On peut donc s'attendre à une dégradation progressive des tissus pulmonaires plus particulièrement exposés aux agressions virales. Cette dégradation peut être la cause d'une susceptibilité accrue aux infections bactériennes qui sont souvent observées chez les individus immuno-déprimés. Par ailleurs, les monocytes/macrophages peuvent présenter des antigènes bac- 
tériens par les molécules de classe I et ce processus, mis en évidence in vitro [7], pourrait être physiologiquement important dans la défense immunitaire au niveau des poumons.

L'ensemble de ces observations pose donc encore de nombreuses questions sur les mécanismes de déf ense immunitaires mis en jeu pour compenser le déficit d'expression en molécules de classe I qui, paradoxalement, s'avère être compatible dans une certaine mesure avec la vie

\section{RÉFÉRENCES}

1. de la Salle H, Hanau D, Fricker D, Urlacher A, Kelly A, Salamero J, Powis SH, Donato $L$, Bausinger $H$, Laforêt $M$, Jeras $M$. Spehner D, Bieber T, Falkenrodt A, Cazenave JP, Trowsdale J, Tongio MM. Homozygous human TAP peptide transporter mutation in HLA class I deficiency. Science 1994 ; 265 : 237-41.

2. Fehling HJ, Swat W, Laplace C, Kühn R, Rajewsky K, Müller U, von Boehmer $H$. MHC Class I expression in mice lacking the proteasome subunit LMP-7. Srience 1994 ; $265: 1234-7$.

3. Bahram S. Transporteurs de peptides et présentation de l'antigène. médecine/sciences $1993 ; 9: 1204-13$.

4. Hanau D, Fricker D, Bieber T, ErpositoFarese ME, Bausinger H, Cazenave JP, Donato L, Tongio MM, de la Salle H. CD1 expression is not affected by human peptide transporter deficiency. Hum Immunol 1194 ; 41:61-8.

5. Schirmbeck R, Reimann J. Peptide transporter-independent, stress protein-mediated endosomal processing of endogenous protein antigens for major histocompatibility complex class I presentation. Eur J Immunol $1994 ; 24$ : 1478-86.

6. Heemels MT, Ploegh H. Untapped peptides. Curr Biol 1993 ; 3 : 380.

7. Pfeifer JD, Wick MJ, Roberts RL, Findlay K, Normark SJ, Harding CV. Phagocytic processing of bacterial antigens for class MHC presentation to T cells. Nature 1993 $361: 359-62$.

Henri de la Salle, Daniel Hanau, Marie-Marthe Tongio

Laboratoire d'histocompatibilité du CRTS de Strasbourg, 10, rue Spielmann, 67085 Strasbourg Cedex, France.

\section{Lionel Donato}

Service de pneumologie pédiatrique de l'hôpital de Hautepierre, Strasbourg.

\section{TIRÉS À PART}

